\title{
The effect of probiotics and phototherapy combined application in comparison with phototherapy alone on bilirubin reduction in term neonates: $A$ Randomized Controlled Trial
}

\section{Ensiyeh Jenabi ( $\nabla$ en.jenabi@yahoo.com )}

Hamadan University of Medical Sciences https://orcid.org/0000-0002-4536-0814

Fatemeh Eghbalian

Hamadan University of Medical Sciences

Mohamad Kazem Sabzehei

Hamadan University of Medical Sciences

Roya Reisi

Hamadan University of Medical Sciences

Soroush Taheri Talesh

Hamadan University of Medical Sciences

\section{Research Article}

Keywords: Neonatal jaundice, phototherapy, probiotics

Posted Date: March 2nd, 2022

DOI: https://doi.org/10.21203/rs.3.rs-1387582/v1

License: (c) (1) This work is licensed under a Creative Commons Attribution 4.0 International License.

Read Full License 


\section{Abstract}

Purpose: This study aimed to evaluate the effect of probiotics and phototherapy combined application in comparison with phototherapy alone on bilirubin reduction in term neonates hospitalized in Iran.

Methods: This clinical trial study included 150 term neonates diagnosed with neonatal jaundice. Patients were equally assigned to two groups of phototherapy with oral probiotics (PediLact drop, 10 drops daily) and phototherapy alone through a simple random sampling method. Serum Bilirubin Levels (SBL) at the time of intervention, 24,48 , and 72 hours later, duration of phototherapy, duration of hospitalization, and the need for blood transfusion were compared in the two groups.

Results: The mean serum bilirubin level (SBL) on the first day of hospitalization was $15.6 \pm 1.7 \mathrm{mg} / \mathrm{dl}$ in the combinatory therapy group, and it was $15.8 \pm 1.6 \mathrm{mg} / \mathrm{dl}$ in the Monotherapy group ( $P=0.584)$. The mean serum level (SBL) on the second day in the combinatory therapy group was $11.2 \pm 2.2$, and in the other group it was $12.4 \pm 2.1$, and on the third day, they were $7.2 \pm 0.9$ and $7.8 \pm 0.7$, respectively, and the difference between the two groups was statistically significant. The mean hospital stay time in the combinatory therapy group was $2.4 \pm 0.5$, and in the phototherapy group, it was $2.8 \pm 0.6$ days $(P=0.001)$. The duration of phototherapy in the combinatory therapy group was $26.2 \pm 9.9$ days, and in the phototherapy group, it was $31.4 \pm 10.3$ hours $(P=0.001)$.

Conclusions: The findings show that the addition of oral probiotics to phototherapy in neonatal jaundice treatment is associated with phototherapy duration reduction and hospital stay time.

\section{Introduction}

Jaundice (icterus) is the most common cause of hospitalization in infancy, especially in the first week after birth. It is also one of the most important causes of neonatal disease [1]. Gestational age less than 37 weeks, history of phototherapy in previous neonates, exclusive breastfeeding, and evident jaundice in the first 24 hours after birth are the most important risk factors for jaundice in neonates [2]. $60 \%$ of fullterm neonates (third day of birth) and $80 \%$ of premature neonates (fifth day of birth) develop jaundice. Jaundice is caused by an increase in direct or indirect bilirubin [3]. Bilirubin is also one of the final products of catabolism. In neonates, due to the short lifespan of erythrocytes compared to adults (90 days vs. 120 days) and due to lack of liver development in bilirubin excretion due to deficiency in glucuronyl-2-transferase, bilirubin production will be higher than excretion, which causes bilirubin deposition in the skin and mucous membranes resulting in physiological jaundice in the neonates $[4,5]$.

Phototherapy is an effective and safe way to reduce indirect bilirubin levels, especially if it starts before the serum bilirubin level rises to the levels that lead to Kernicterus. Phototherapy uses white and blue light with a wavelength of 420-450, which effectively reduces bilirubin levels. Under the influence of light, bilirubin is converted into water-soluble isomers and will be excreted. However, it has side effects such as retinal injury, hyperthermia, dehydration, loose stools (diarrhea), and Bronze baby syndrome [6, 7]. If neonates with jaundice are not treated, some of them may have persistent traces due to jaundice. Drugs 
used to treat non-conjugated neonatal jaundice include Phenobarbital, Metalloporphyrins, oral charcoal, clofibrate [8-10], and D-penicillamine, requiring further studies to confirm their safety [11]. Probiotics are microorganisms that can reduce the transport time of substances in the intestine [12]. Few studies have shown the positive effects of probiotics on lowering serum bilirubin levels. These studies have shown to some extent that probiotic compounds reduce the time duration required for phototherapy [13-15]. Therefore, in this study, we investigated the effect of probiotic compounds on serum bilirubin levels (SBL) and the duration needed for phototherapy in term neonates.

\section{Materials And Methods}

Study design and setting

This was a parallel, randomized controlled trial performed for eight months (from 23 October 2019 to 20 June 2020) in a pediatric hospital in western Iran. Before entering the study, informed consent was obtained from the parents of the neonates or their legal guardians. The ethics committee of Hamadan University of Medical Sciences was registered with the ID IR.UMSHA.REC.1398.458 and in the Iranian Clinical Trial Database (IRCT) with the ID IRCT20120215009014N306.

Study population

Inclusion criteria were full-term neonates (37 to 42 weeks), weight over $2500 \mathrm{~g}$ at birth, neonates with no abnormalities, and seemingly healthy. Neonates with blood group incompatibility, Rh incompatibility, glucose 6-phosphate dehydrogenase deficiency, first-day jaundice, and neonates who received drugs such as Phenobarbital during pregnancy by the mother and previous treatment of neonatal jaundice with medication or home treatment were excluded. The sample size was estimated using the findings of previous studies [10]. The average bilirubin level in the control and intervention groups was 15 and 13 $\mathrm{mg} / \mathrm{dl}$, respectively. Standard deviation was 2 and $90 \%$ power and $10 \%$ loss. Finally, 75 people for each group were estimated.

Randomization

Randomization was performed using softwarerandomization.com. For allocation concealment, the randomization instructions were given to the investigator and research assistants in sequentially numbered, opaque, sealed, identical envelopes (SNOSE) with an unpredictable allocation code.

Study interventions

The intervention group was treated with standard phototherapy with probiotics (PediLact drops made by Zist-Takhmir Company; ten daily drops, taken orally). Phototherapy was performed with a device manufactured by Parsa Company with 5 special blue lamps and $420-450 \mathrm{~nm}$ wavelength, which was placed $30 \mathrm{~cm}$ above the neonate. We used billitest $A$ for measuring total bilirubin. The control group only received phototherapy. In present study, clinicians, caregivers and the laboratory staff performing 
analysis of blood bilirubin levels were blinded but, the study staff and the baby were not due to the nature of the intervention.

Outcome measures

The blood bilirubin levels were measured at the start of treatment and 24,48 , and 72 hours after that. All neonates were examined on an outpatient basis for jaundice, general condition, and possible complications 2 days after discharge in Besat Hospital.

\section{Data collection}

Information of participating neonates was completed using a checklist including information on mother's age, neonate age, neonate weight, gestational age, neonate sex, and recorded bilirubin levels at the start of treatment, 24,48 , and 72 hours after treatment, duration of phototherapy, and the length of hospital stay. The validity and reliability of the checklist were also evaluated. 10 pediatricians confirmed the validity of the checklist, and their opinions were applied.

\section{Statistical analysis}

SPSS24 software was used to analyze the data. Mean and standard deviation were used to describe quantitative variables; frequency and frequency percentages were used for qualitative variables. The Kolmogorov-Smirnov test showed that the studied quantitative variables had a normal distribution. Therefore, $\mathrm{t}$-test and repeated analysis of variance were used to compare quantitative variables. The significance level was less than 0.05 .

\section{Results}

In this clinical trial study, 150 term neonates with a diagnosis of jaundice meeting the inclusion criteria were divided into two groups: phototherapy with oral probiotics (combinatory therapy) and phototherapy (monotherapy) alone. There was no statistically significant difference between the two groups regarding gender, gestational age, mother's age, neonate age at the time of hospitalization, and birth weight. In this respect, both groups were similar in terms of variables (Table 1). The hospitalization duration and phototherapy duration of neonates in combinatory therapy were statistically and significantly lower (Table 2). None of the examined neonates required blood transfusion. Serum bilirubin levels were comparable at the start of treatment in both groups. On the second and the third day after the intervention, serum bilirubin levels decreased in both groups, which was more significant in the combinatory therapy group (Table 3). Repeated analysis of variance test showed that the bilirubin rate in both groups was significantly lower than the rate recorded at the beginning of the study (Table 4). 
Table 1

Comparison of basic features in both groups

\begin{tabular}{|c|c|c|c|}
\hline Variable & $\begin{array}{l}\text { Combinatory } \\
\text { treatment } \\
\mathrm{N}=75\end{array}$ & $\begin{array}{l}\text { Phototherapy } \\
\mathrm{N}=75\end{array}$ & $\begin{array}{l}\mathrm{P}- \\
\text { value }\end{array}$ \\
\hline Gender of neonate & $43(57.3)$ & $39(52.0)$ & 0.512 \\
\hline Frequency & $32(42.7)$ & $36(48.0)$ & \\
\hline Gestational age (week), mean \pm sd & $37.8 \pm 0.8$ & $37.6 \pm 0.7$ & $0.118 ¥$ \\
\hline Mother's age (year), mean \pm sd & $26.0 \pm 2.6$ & $25.7 \pm 2.5$ & $0.413 ¥$ \\
\hline $\begin{array}{l}\text { Neonate' age at the time of hospitalization } \\
\text { (day), } \\
\text { mean } \pm \text { sd }\end{array}$ & $3.9 \pm 1.3$ & $4.0 \pm 1.2$ & $0.611 ¥$ \\
\hline Weight of the neonate at birth, mean \pm sd & $3097 \pm 329.2$ & $3062 \pm 300.3$ & $0.512 ¥$ \\
\hline
\end{tabular}

Table 2

Comparison of the length of the hospital stay and the duration of phototherapy of both groups

\begin{tabular}{|c|c|c|c|}
\hline \multirow[t]{3}{*}{ Treatment groups } & Combinatory treatment & Monotherapy & \multirow[t]{3}{*}{ P-value } \\
\hline & $N=75$ & $N=75$ & \\
\hline & Mean \pm SD & Mean \pm SD & \\
\hline the length of the hospital stay (day) & $2.4 \pm 0.5$ & $2.8 \pm 0.6$ & 0.001 \\
\hline the duration of phototherapy (hour) & $26.2 \pm 9.9$ & $31.4 \pm 10.3$ & 0.001 \\
\hline
\end{tabular}

Table 3

Mean serum bilirubin level in $\mathrm{mg} / \mathrm{dL}$ in the two groups, measured at three intervals

\section{Time of measurement}

Combinatory treatment

$$
\mathrm{N}=75
$$

Mean \pm SD

$15.6 \pm 1.7$

$11.2 \pm 2.2$

$7.2 \pm 0.9$
Phototherapy

$N=75$

Mean \pm SD

$15.8 \pm 1.7$

$12.4 \pm 2.1$

$7.8 \pm 0.7$

The third day

The number of patients on the third day in the combinatory treatment group and the phototherapy group was 31 and 53, respectively. 
Table 4

Analysis of variance test with the repeated rate of bilirubin in both groups

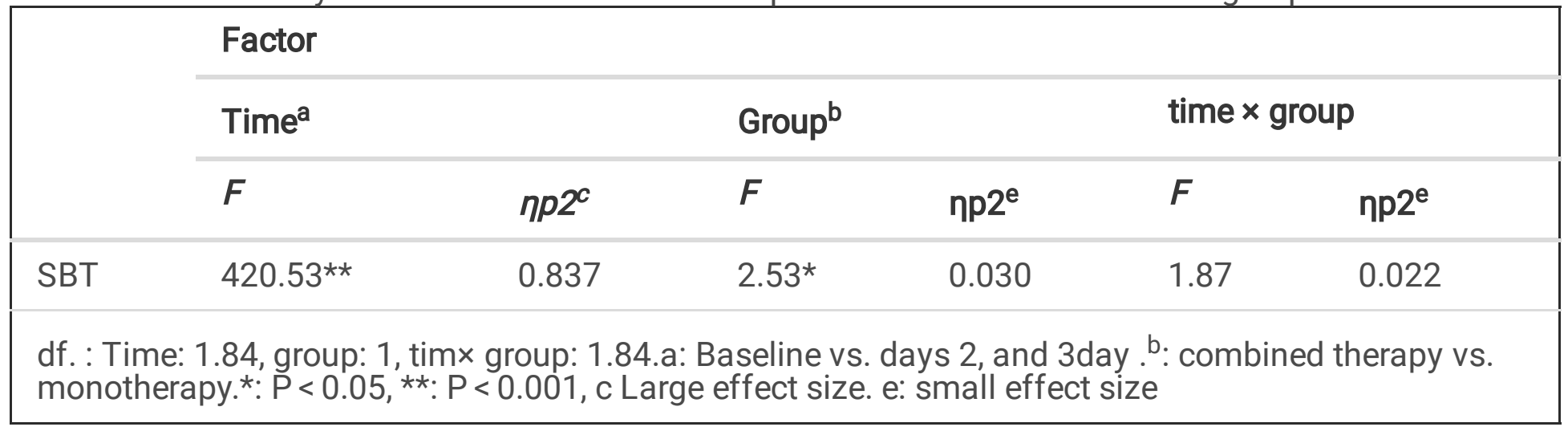

\section{Discussion}

According to the present study's findings, probiotics as an adjuvant treatment with phototherapy in neonatal jaundice treatment can reduce the length of hospital stay and the phototherapy duration that reduces the costs and the separation time of neonates from mothers.

A slight increase in bilirubin levels usually has no severe side effects other than jaundice and, in most cases, is not dangerous, so it does not require special treatment [2]. However, neonatal jaundice can be a concern for parents and increase hospital costs due to hospitalization. Therefore, proper and timely diagnosis and treatment of Hyperbilirubinemia is crucial. Current recommendations for neonatal hyperbilirubinemia management focus on determining the level of specific bilirubin in infancy before initiating phototherapy. Hyperbilirubinemia occurs in approximately two-thirds of neonates in the first days of life and is often treated by phototherapy. Although this is a safe procedure, there is a concern that it could potentially cause DNA damage and severe side effects, especially in premature neonates. Researchers have found that Hyperbilirubinemia does not cause DNA damage and apoptosis, while phototherapy (both normal and severe) is associated with DNA damage in neonates [16, 17]. Also, phototherapy may lead to early complications such as abnormalities of the neonate's body temperature, water and electrolytes, Bronze Baby syndrome, retinal damage and skin injuries, and in some cases, late complications such as neoplasm and allergic diseases due to the separation of the baby from the mother $[18,19]$. Therefore, other alternative therapies instead of phototherapy have been considered in recent years, and several pharmacological methods, including the use of clofibrate in the treatment of neonatal jaundice, have been introduced for this purpose in studies of Eghbalyian et al. [8, 9, 20] However, studies on the use of probiotics in the treatment of neonatal jaundice are controversial.

Most studies have shown that probiotics effectively decrease serum bilirubin levels (SBL). The findings of a recent study by Ahmadipour et al. [6] in Khorramabad city, Lorestan province, showed that adding 5 drops per day of probiotics (synbiotics) compared to the control group (phototherapy) on 83 term neonates could significantly decrease the length of hospital stay. In this study, fecal and urinary excretion per day significantly increased in the intervention group, which was not studied in the present study. Demirel et al. [15] examined the effect of the probiotic Saccharomyces Boulardii on 179 neonates with 
very low birth weight and jaundice. Their findings showed that the phototherapy duration in neonates who received probiotics was shorter than in the control group. In the present study, no statistically significant difference was observed between the SBL of the two groups. Yuan et al. [21] also evaluated the oral effect of probiotics and their safety in treating Hyperbilirubinemia in neonates with jaundice. They concluded that additional treatment with probiotics might increase the effect of phototherapy and the level of immunity in neonates. In a clinical trial study, Mu et al. [22] investigated the effect of Saccharomyces Boulardii on serum bilirubin levels. They found that Saccharomyces Boulardii is effective in reducing serum bilirubin levels. Armanian et al. [13] examined the effect of prebiotics on jaundice of 50 premature neonates. In this study, oligosaccharides were used as probiotics. According to their findings, following prebiotics application, feces per day and serum bilirubin levels decreased. In the present study, fecal excretion per day was not studied, which was one of the limitations of our study. Lingling et al. [23] also observed in their study that the probiotic Mami reduces jaundice following exclusive breastfeeding by increasing the intestinal flora in neonates. Bisceglia et al. studies 76 neonates and reported bilirubin levels in neonates who received probiotics for 72 hours were significantly lower than in controls. In this study, bilirubin was measured using dermal bilirubin. Torkaman et al.'s [24] study findings were consistent with the present study and showed that adding probiotics to phototherapy has a good effect on reducing the length of hospital stay in neonates and is not associated with any specific side effects.

Contrary to the results of this study, Zahed Pasha et al. [25] studied 150 term neonates with jaundice in Babol city, Mazandaran province, in 2017. 75 neonates received probiotics in addition to phototherapy; decreased serum bilirubin levels and duration of phototherapy were not significantly different between the two groups. Another study by Serce et al. [26] on 119 neonates showed that the probiotic Saccharomyces combined with phototherapy had no significant effect on reducing SBT and the duration of phototherapy.

Some reasons for the differences in the studies were the type of probiotic used, the method of following up, measuring the serum bilirubin level, and the sample (term, non-term), as well as the characteristics of the patients. Probiotics are beneficial live bacteria found in certain foods or supplements. They can provide many health benefits, while prebiotics are the foods that probiotics feed on. Probiotics have a beneficial effect on the intrahepatic cycle, including better gastrointestinal motility, improving and increasing the excretion, and viscosity of feces may help reduce bilirubin and excretion [27].

In this study, none of the neonates who received probiotics and the neonates who received phototherapy needed blood transfusions. They also did not develop serious complications such as encephalopathy. In recent years, due to advances in the treatment of these patients, blood transfusion in these neonates is rarely necessary. Findings of studies, including the meta-analysis study by Armanian et al. [13], which recently reviewed clinical trial studies, showed that probiotics do not have any significant side effects in the treatment of neonatal jaundice. In the present study, no severe side effects were observed following the use of probiotics. The findings of this study suggest that the combined application of phototherapy and probiotics is effective in reducing bilirubin levels, which ultimately reduces hospital stay length and 
the duration of phototherapy than phototherapy alone. Performing similar studies in other areas and regions are suggested.

\section{Conclusion}

According to the findings of this study, although both phototherapy alone and phototherapy combined with probiotics are effective in reducing serum bilirubin levels in term neonates with jaundice, given that the length of hospital stay and the duration of phototherapy in neonates receiving probiotics are shorter, this treatment method is preferred in these neonates. However, more studies are needed to confirm the results of this study and other performed studies.

\section{Declarations}

\section{Conflict of interest}

The authors had no conflict of interest in conducting the study.

\section{Acknowledgements}

We would like to thank the Hamadan University of Medical Sciences for the support of this study.

\section{Funding}

This study supported from the Hamadan University of Medical Sciences.

\section{Conflict of interest}

The authors report no conflict of interest.

\section{Availability of data and material:}

Data given in tables and as text in manuscript.

\section{Code availability:}

Not applicable

\section{Authors' contributions}


Conceptualization: Eghbalian F, Sabzehei M, Jenabi E, Taheri S, Reisi R

Data curation: Eghbalian F, Sabzehei M, Jenabi E, Taheri S, Reisi R

Methodology: Eghbalian F, Sabzehei M, Taheri S

Funding acquisition: Eghbalian $\mathrm{F}$

Software: Jenabi E

Writing - original draft: Jenabi E, Eghbalian F, Taheri S

Writing - review \& editing: All authors

\section{Ethics approval:}

informed consent was obtained from the parents of the neonates or their legal guardians. The ethics committee of Hamadan University of Medical Sciences was registered with the ID IR.UMSHA.REC.1398.458 and in the Iranian Clinical Trial Database (IRCT) with the ID IRCT20120215009014N306.

\section{Consent to participate:}

informed consent was obtained from the parents of the neonates or their legal guardians.

\section{Consent for publication:}

Not applicable

\section{References}

1. Wan ASL, Daud SM, Teh SH, Choo YM, Kutty FM (2016) Management of neonatal jaundice in primary care. Malaysian family physician: the official journal of the Academy of Family Physicians of Malaysia 11(2-3):16

2. Mitra S, Rennie J (2017) Neonatal jaundice: aetiology, diagnosis and treatment. Br J Hosp Med 78(12):699-704

3. Ambalavanan N, Carlo WA (2011) Jaundice and Hyperbilirubinemia in the newborn. In: Kliegman RM, Stanton BF, St. Geme JW, Schor NF, Behrman RE (eds). Ed t, editor:Philadelphia: Elsevier Saunders;

4. Chee Y, Chung PH, Wong RM, Wong KK (2018) Jaundice in infants and children: causes, diagnosis, and management. Hong Kong Med J 24(3):285-292 
5. Jirsa M, Sticová E (2013) Neonatal hyperbilirubinemia and molecular mechanisms of jaundice. Vnitr Lek 59(7):566-571

6. Ahmadipour S, Baharvand P, Rahmani P, Hasanvand A, Mohsenzadeh A (2019) Effect of synbiotic on the treatment of jaundice in full term neonates: a randomized clinical trial. Pediatr Gastroenterol Hepatol Nutr 22(5):453

7. Dennery PA (ed) (2002) editor Pharmacological interventions for the treatment of neonatal jaundice. Seminars in neonatology. Elsevier

8. Eghbalian F, Jenabi E, Hatami E, Basiri B, Derakhshandeh K, Pezeshki N et al (2021) Clofibrate in treatment of the non-hemolytic hyperbilirubinemia in preterm neonates in the west of Iran.Iranian Journal of Neonatology IJN.

9. Eghbalian F, Monsef F, Alam Ghomi N, Monsef A (2013) Effect of low versus moderate dose of clofibrate on serum bilirubin in healthy term neonates with indirect hyperbilirubinemia. Iran J Med Sci 38(4):349-350

10. Kumar P, Adhisivam B, Bhat BV (2017) Clofibrate as an Adjunct to Phototherapy for Unconjugated Hyperbilirubinemia in Term neonates. Indian J Pediatr 84(10):763-767

11. Kutz K, Kandler H, Gugler R, Fevery J (1984) Effect of clofibrate on the metabolism of bilirubin, bromosulphophthalein and indocyanine green and on the biliary lipid composition in Gilbert's syndrome. Clin Sci 66(4):389-397

12. Husebye E, Hellström PM, Sundler F, Chen J, Midtvedt T (2001) Influence of microbial species on small intestinal myoelectric activity and transit in germ-free rats. Am J Physiology-Gastrointestinal Liver Physiol 280(3):G368-G80

13. Armanian AM, Barekatain B, Hoseinzadeh M, Salehimehr N (2016) Prebiotics for the management of hyperbilirubinemia in preterm neonates. J Maternal-Fetal Neonatal Med 29(18):3009-3013

14. Armanian AM, Jahanfar S, Feizi A, Salehimehr N, Molaeinezhad M, Sadeghi E (2019) Prebiotics for the prevention of hyperbilirubinaemia in neonates.Cochrane Database of Systematic Reviews. ;(8).

15. Demirel G, Celik IH, Erdeve O, Dilmen U (2013) Impact of probiotics on the course of indirect hyperbilirubinemia and phototherapy duration in very low birth weight infants. J maternal-fetal neonatal Med 26(2):215-218

16. Yahia S, Shabaan AE, Gouida M, El-Ghanam D, Eldegla H, El-Bakary A et al (2015) Influence of hyperbilirubinemia and phototherapy on markers of genotoxicity and apoptosis in full-term infants. Eur J Pediatrics 174(4):459-464

17. Ramy N, Ghany E, Alsharany W, Nada A, Darwish R, Rabie W et al (2016) Jaundice, phototherapy and DNA damage in full-term neonates. J Perinatol 36(2):132-136

18. Ebbesen F, Hansen TW, Maisels MJ (2017) Update on phototherapy in jaundiced neonates. Curr Pediatr reviews 13(3):176-180

19. Faulhaber FR, Procianoy RS, Silveira RC (2019) Side effects of phototherapy on neonates. Am J Perinatol 36(03):252-257 
20. Eghbalian F, Pourhossein A, Zandevakili H (2007) Effect of clofibrate in non-hemolytic indirect hyperbilirubinemia in full term neonates. Indian J Pediatr 74(11):1003-1006

21. Yuan C, Chen J, Lu C (2011) Efficacy of oral probiotics and its effect on immunity in treating hyperbilirubinemia of neonates. Jiangsu Med J 2:018

22. Yu M-x, Chen D-p, Yin Z-j (2005) LIU Y-x. The effect of probiotics on the incidence of neonatal hyperbilirubinemia.Chin J Microecol.

23. Ling-ling W (2006) Fen-lan B. A Clinical Observation on Effect of" Mamiai" on Lowering Breast Milk Jaundice by Quickly Constructing Intestinal Microflora [J].Clinical Journal of Medical Officer. ; 4

24. Torkaman M, Mottaghizadeh F, Khosravi MH, Najafian B, Amirsalari S, Afsharpaiman S (2017) The effect of probiotics on reducing hospitalization duration in infants with hyperbilirubinemia.Iranian Journal of Pediatrics. ; 27(1)

25. Zahed Pasha Y, Ahmadpour-Kacho M, Ahmadi Jazi A, Gholinia H (2017) Effect of Probiotics on Serum Bilirubin Level in Term Neonates with Jaundice; A Randomized Clinical Trial. Int J Pediatr 5(10):5953-5958

26. Serce O, Gursoy T, Ovali F, Karatekin G (2015) Effects of Saccharomyces boulardii on neonatal hyperbilirubinemia: a randomized controlled trial. Am J Perinatol 32(02):137-142

27. Liu W, Liu H, Wang T, Tang X (2015) Therapeutic effects of probiotics on neonatal jaundice. Pakistan J Med Sci 31(5):1172

\section{Figures}




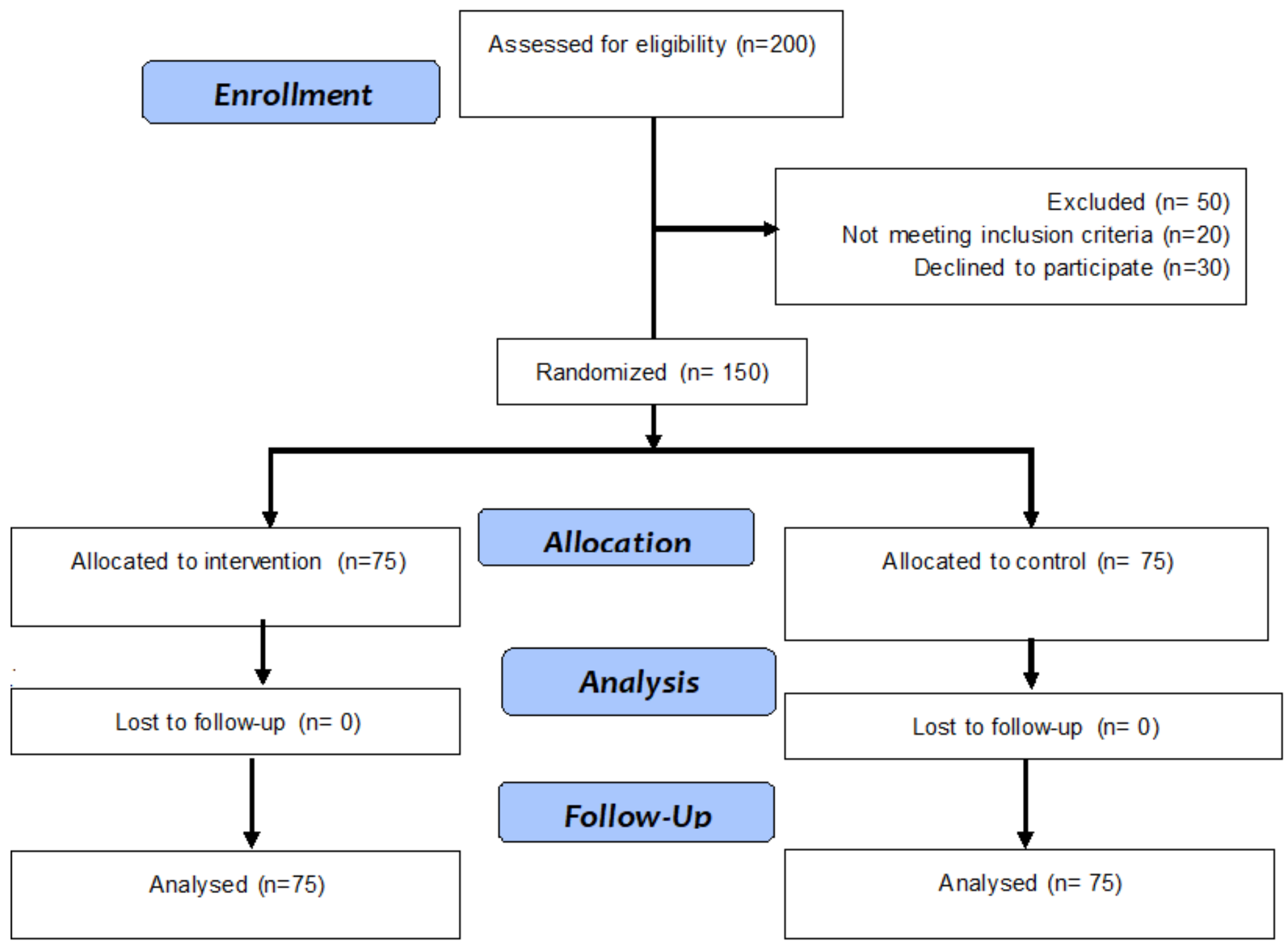

Figure 1

Please See image above for figure legend. 